\title{
A Physical Chemistry Study of Black Powder Materials by Solution Combustion Synthesis Method
}

\author{
Fitria Hidayanti ${ }^{1}{ }^{*}$, Kiki R. Lestari ${ }^{1}$, Nano Sujani $^{1}$, Jarot Raharjo ${ }^{2}$ \\ ${ }^{1}$ Engineering Physics Department, Universitas Nasional, Jakarta Selatan 12520, Indonesia. \\ ${ }^{2}$ Center for Materials Technology, Agency for the Assessment and Application of Technology Puspiptek Building No. 224, \\ South Tangerang, Banten 15314, Indonesia. \\ Corresponding author* \\ fitriahidayanti@gmail.com
}

Manuscript received: 14 August 2021. Revision accepted: 30 September, 2021. Published: 08 October, 2021.

\begin{abstract}
A study on the synthesis of black powder $\left(\mathrm{La}_{2} \mathrm{NiO}_{4}\right)$ material using the solution combustion synthesis method at a variation of synthesis temperature of 60,70 , and $80^{\circ} \mathrm{C}$ was carried out. It produces a mass of black powder of 2 grams by four times of synthesis process. Then, material characterization was performed on the black powder samples obtained by using X-ray Diffraction (XRD) to determine the phases formed, Scanning Electron Microscopy - Energy Dispersive X-ray Spectroscopy (SEM-EDS) to determine the morphology and analyze the composition elemental on the microscale and Fourier Transform Infra-Red (FTIR) to determine chemical bonds. From the whole black powder sample, XRD analysis showed the phases of Dilantanum Nickel Tetraoxide $\left(\mathrm{La}_{2} \mathrm{NiO}_{4}\right)$, Nickel Oxide (NiO), Lanthanum Oxide $\left(\mathrm{La}_{2} \mathrm{O}_{3}\right)$, and Lanthanum Oxide $\mathrm{Ht} \mathrm{x}$-form $\left(\mathrm{La}_{2} \mathrm{O}_{3} \mathrm{Ht}(\mathrm{x}\right.$-form $)$ ). In addition, it was seen from the visible compositions of the phases that the $\mathrm{NiO}$ phase looks more dominant and the variation of the synthesis temperature shows that the $\mathrm{La}_{2} \mathrm{O}_{3}$ phase was increasing. This was supported by the EDS analysis, which showed that the EDS spectrum contains elements $\mathrm{La}, \mathrm{Ni}$, and $\mathrm{O}$ where the element $\mathrm{O}$ indicates that oxidation occurs in the elements $\mathrm{Ni}$ and La. On the other hand, the SEM analysis results confirm that the black powder sample contains the elements $\mathrm{La}$ and $\mathrm{Ni}$, based on the high and low electron images contained in the morphology of the black powder sample. In addition, it was also known that the particles in the black powder sample were micron size and had porous morphology. This occurs due to rapid thermal decomposition events and excessive gas development. In addition, FTIR analysis showed that the O-H bond had been reduced and there are still $\mathrm{C}-\mathrm{O}$ and $\mathrm{C}-\mathrm{H}$ bonds indicating the presence of organic elements possessed by glycine.
\end{abstract}

Keywords: $\mathrm{La}_{2} \mathrm{NiO}_{4}$, Solution Combustion Synthesis; Battery NiMH; Hydrogen Storage Alloy.

\section{INTRODUCTION}

The development of electronic technology and electric cars can not be separated from the role of energy sources as a power to use these technologies. One source of energy booster is the battery. A battery is a device that has a working principle to convert chemical energy into electrical energy through electrochemical processes. One fairly rapid development occurred in the type of rechargeable batteries. A rechargeable battery is a type of battery that can be recharged again with the Charge \& Discharge System. Similar to the type of primary battery, batteries generally consist of 3 main components, namely the anode, cathode, and electrolyte.

There are very rapid development in Nickel Metal Hydride (NiMH) batteries (Chang et al., 2016, Young et al., 2017). Highpower International Inc., which is a clean energy supplier company integrated with Research and Development, manufacturing and sales of NiMH and Li-Ion rechargeable batteries, as well as energy storage and battery recycling systems, explains that the majority of the $74 \%$ NiMH battery consumer market share in China, followed by Japan as $14 \%$ and Europe as $10 \%$, and $2 \%$ are the others. In addition, European countries still have a large market level for the consumption of NiMH batteries. For example, in 2013, Germany, the Netherlands, Belgium, and the UK accounted for $8.3 \%$ of NiMH battery needs from China, and in February 2014, North America received 51.76\% of NiMH battery needs, followed by Europe receiving $32.7 \%$, and $13.73 \%$ (Chang et al., 2016). The data shows that the market share of NiMH batteries is still growing, and Indonesia may still be able to develop NiMH batteries given the abundant natural resources for raw materials for NiMH batteries.

Indonesia is a country that has the second-largest wealth of nickel in the world. Nickel ore is contained in nickel oxides or laterites, which are spread in Eastern Indonesia (Prasetiyo, 2008). Based on data from the Geological Resource Center in 2016, Indonesia has nickel resource potential of 5,756,362,683 (ore) and $79,172,702$ (metals) and nickel reserves of $3,197,178,940$ (ores) and 50,872,304 (metals) with elemental content nickel average $1.20-3.25 \%$. These 
nickel resources and reserves in 2011-2015 continued to increase due to the discovery of new nickel resources (Haryadi, 2017). In contrast, the potential of soil metals is rarely found in monazite and xenotime minerals with reserves of more than 951,000 tons (Inatadon et al., 2015). Some of Indonesia's rare earth metal minerals are contained in an associated tin in Bangka Belitung and gold in Kalimantan (Virdhian and Afrilinda, 2014). Both of these minerals are raw materials from NiMH batteries.

NiMH batteries are secondary batteries in which Metal Hydride acts as a negative electrode and hydrogen-absorbing alloy acts as an active substance in the negative anode. The development of NiMH batteries received considerable attention from researchers, especially their use in Hybrid Electrical Vehicles (HEV) technology. In addition, the unique characteristics of the working principle of the battery, especially in the Hydrogen Storage Alloy as the anode of the battery, is an interesting topic for writers and has been developed by several researchers, such as research conducted by several institutions in China, Japan, Europe, and America (Chang et al., 2016, Young et al., 2017, Ouchi et al., 2016, Chang, et al., 2017). The research mainly focuses on NiMH battery anode components, where there is a Hydrogen Storage Alloy material. The role of hydrogen storage alloy material in this case are greatly affects to NiMH battery power density. Furthermore, its role is very important in the absorption/adsorption process of $\mathrm{H}_{2}$ at negative electrodes.

\section{Nickel Metal Hydride (NiMH) Batteries}

NiMH batteries are rechargeable batteries by utilizing electrochemical reactions found in active materials or electrodes. Like most batteries, the main components in $\mathrm{NiMH}$ batteries are the same as batteries in general, consisting of negative and positive electrodes and electrolyte solutions. NiMH battery's active material used on the positive electrode (cathode) is $\mathrm{Ni}(\mathrm{OH})_{2}$. At the same time, the hydrogen storage alloy material is used as active material on negative electrodes. In addition, the electrolyte solution that is usually used in NiMH batteries is potassium hydroxide $(\mathrm{KOH})$. In NiMH battery cathode an electrochemical reaction (Equation (1)) is occur as follows:

$$
\mathrm{Ni}(\mathrm{OH})_{2}+\mathrm{OH}^{-} \rightarrow \mathrm{NiOOH}+\mathrm{H}_{2} \mathrm{O}+e^{-}
$$

In the charging process, atoms in Nickel Hydroxide $\left(\mathrm{Ni}(\mathrm{OH})_{2}\right)$ compounds will dissociate to form Nickel Oxide Hydroxide (NiOOH) compounds and water and electrons. Then the hydrogen atom moves through the diaphragm (electrolyte) to the NiMH battery anode in combination with the metal hydride material. Meanwhile, electrons flow to the anode through an external circuit, then the reaction occurs (Equation (2)) in the anode as follows:
$\mathrm{M}+\mathrm{H}_{2} \mathrm{O}+e^{-} \rightarrow \mathrm{MH}+\mathrm{OH}^{-}$

Then the charging process at the cathode occurs where an HSA material and a hydrogen atom dissociate at the cathode and an electron to form a Metal Hydride material and a Hydroxide ion. Briefly, the process of charging and discharging is shown in Figure 1.

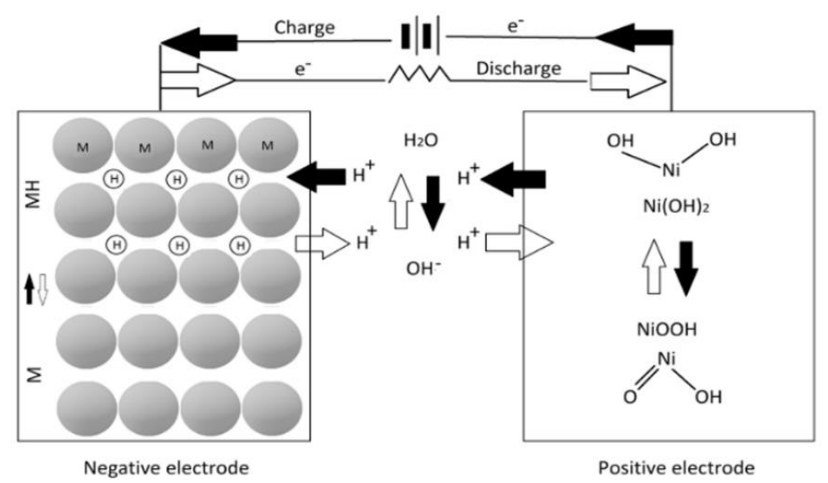

Figure 1. Charge and discharge mechanism on Nickel Metal Hydride batteries (Chang, S., Young, K. H., \& Lien, Y. L., 2017).

\section{Solution Combustion Synthesis Method}

Solution Combustion Synthesis (SCS) is an effective method for the synthesis of nanoscale materials, versatile and efficient in the use of energy that has been used in the production of various types of Ceramic Powders for several types of advanced applications ( $\mathrm{Li}$ et al., 2016, Khina, 2010). The SCS method is a simple method but an important technique in synthesising and processing nanostructures of metal, oxide, spinel, alloy, and intermetallic (Xanthopoulou et al., 2018). This method is based on an exothermic reaction between nitrates (oxidizing agents) and organic substances (reducing agents) by directly producing nanostructured powders (Xanthopoulou et al., 2018, Deganello et al., 2018). Utilization of the exothermic reaction as a source to drive the reaction itself so that no external source is needed and only requires ignition temperature to start the process (González-Cortés et al., 2013). Crucial advantages of the SCS method are (i) mixing of the initial reaction that occurs in a liquid state, thereby facilitating control of the composition, structure, homogeneity, and stoichiometry of the reaction product; (ii) the possibility of incorporating impurity/dopant ions in the form of oxides, to prepare materials that are suitable for industrial needs; (iii) the very fast SCS method allows the formation of metastable phases (Xanthopoulou et al., 2018, Aruna, 2017, Manukyan, 2017). In addition, the main thing that is important from the use of the SCS method is that it can be used with various precursors, both soluble and non-dissolved (Deganello et al., 2018). 


\section{Material Characterization}

Material characterization is an activity carried out to determine the physical properties, mechanical properties, and chemical properties through various tests. Among the tests are Thermogravimetry Analysis (TGA), Differential Thermal Analysis (DTA), DSC, Fourier Transform Infra-Red (FTIR), X-Ray Diffraction (XRD), X-Ray Fluorescence (XRF), Scanning Electron Microscopy (SEM), Energy Dispersive X-Ray Spectroscopy (EDS), UV-Vis, and many other tests. This study, it was conducted characteristic tests on three main types of tests, including (1) X-Ray Diffraction Spectroscopy (XRD), (2) Fourier Transform Infrared Spectroscopy (FTIR), and (3) Scanning Electron Microscope (SEM).

Therefore, this research aimed to obtain Hydrogen Storage Alloy material which is still in the scope of research in black powder synthesis with variations in temperature of synthesis/reaction using the Solution Combustion Synthesis (SCS) method. Then the material characterization analysis was carried out, including crystal size, chemical bonds, functional groups, and morphology on the influence of temperature.

\section{RESEARCH METHODS}

\section{Black Powder Material Synthesis}

Black powder synthesis was carried out by dissolving the three precursors as presented in the synthesis process flow in Figure 2. The precursors used in this synthesis process include 0.433 grams $(1 \mathrm{mmol})$ Lanthanum Nitrate Hexahydride $\left(\mathrm{La}\left(\mathrm{NO}_{3}\right)_{3} \cdot 6 \mathrm{H}_{2} \mathrm{O}\right)$ p.a. which produced by Merck, 1.454 grams $(5 \mathrm{mmol})$ Nickel Nitrate Hexahydride $\left(\mathrm{Ni}\left(\mathrm{NO}_{3}\right)_{2} .6 \mathrm{H}_{2} \mathrm{O}\right)$ p.a. produced by Merck, and 0.976 grams $(7.2 \mathrm{mmol})$ Glycine $\left(\mathrm{C}_{2} \mathrm{H}_{5} \mathrm{NO}_{2}\right)$ is produced by Merck. Then, it is dissolved at $10 \mathrm{~mL}$ distilled water which is equivalent to 10 grams (Adziimaa et al., 2013). After that, simultaneously stirring using magnetic stirring with a total mass of 12.863 grams.

The temperature variations carried out in the stirring process were at temperatures 60,70 and $80{ }^{\circ} \mathrm{C}$ (Xanthopoulou et al., 2018, Liu et al., 2016, Patil et al., 1997, Xanthopoulou et al., 2017). When the stirring process reached the desired temperature, the temperature of the solution was stabilized at a set point at 60,70 , and $80^{\circ} \mathrm{C}$. After that, the transparent green solution that had been carried out in the stirring process on the beaker glass is transferred into the cup for further drying process which aims to remove the formed water element. This drying process was carried out for 3 hours 20 minutes until the gel was formed (Liu et al., 2016). The purpose of forming the gel from the solution between the fuel and the oxidant was tightly connected in the gel network (Deganello et al., 2018).

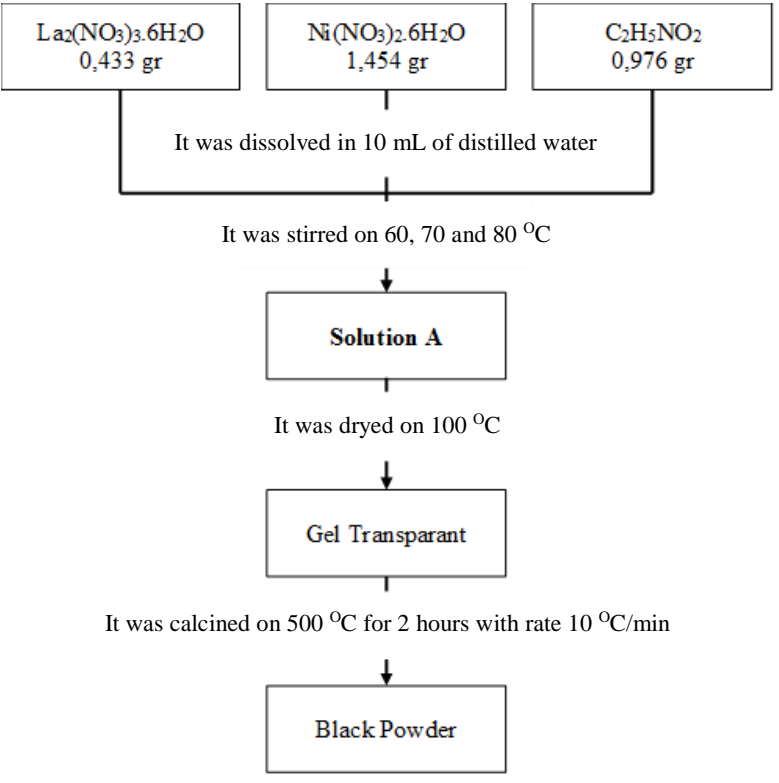

Figure 2. The flow of black powder material synthesis.

The gel obtained from the drying process was then calcined by heating the powder at high temperatures but still under the melting point (Latif et al., 2014). In addition, this process aimed to eliminate volatile elements, heat decomposition and form new phases of the three precursors used. The condition of this gel calcination with a temperature of $500{ }^{\circ} \mathrm{C}$ and a heating rate of $10^{\circ} \mathrm{C} / \mathrm{min}$ is then held for 2 hours (Liu et al., 2016). Then the gel would reach ignition temperature to undergo combustion and change into a sponge shape with volume development. The expanded sample was then mashed using a mortar to obtain a sample in the form of powder.

\section{X-Ray Diffraction}

This process was carried out on the X-Ray Diffraction characterization at the LIPI Physics Research Center with the XRD SmartLab, Rigaku. XRD characterization was carried out under test conditions with a voltage of $40 \mathrm{kV}$ and a current of $30 \mathrm{~mA}$. The target element used to radiate the sample is $\mathrm{Cu} \mathrm{Ka}$ with wavelength specifications $0.1541862 \mathrm{~nm}$. Besides the STEP mode scan with a scan speed of 0.25 and a scan range of 10 $90 \mathrm{deg}$ for each sample. Then an analysis of the X-ray diffraction pattern of the black powder sample was conducted using the Rietveld method using PANalytical High Score Plus software.

\section{SEM-EDS}

The Scanning Electron Microscopy characterization has been carried out at the BPPT Technology Incubator Center using the Scanning Electron Microscope (SEM) type Quanta 650 to test three samples with magnifications of 500,1000, 1500 and 10000 and the Energy Dispersive X-Ray Spectroscopy (EDS) of OXFORD Instruments. 


\section{Fourier Transform Infrared (FTIR)}

Fourier Transform Infrared (FTIR) characterization has been conducted at the Material Technology Center BPPT. The FTIR test was carried out using the Nocolet iS50 Thermo Scientific type FTIR under the conditions of the spectra scan speed test per second 65 (at $16 \mathrm{~cm}^{-1}$ ) and $95\left(\right.$ at $\left.32 \mathrm{~cm}^{-1}\right)$. With a scan range of $400-4000 \mathrm{~cm}^{-1}$ for each black powder sample.

\section{RESULT AND DISCUSSION}

\section{X-Ray Diffraction}

The diffraction pattern of the sample for black powder with a variation of synthesis temperature at $60{ }^{\circ} \mathrm{C}, 70{ }^{\circ} \mathrm{C}$ and $80^{\circ} \mathrm{C}$ is shown in Figure 3. It is known that in the angular range of 10 to 90 degrees, there is an identical diffraction peak pattern of the four black powder samples, the identical peak pattern in the 2theta angles, including $37^{\circ}, 43^{\circ}, 62^{\circ}, 75^{\circ}$ and $79^{\circ}$ which are identified as phases of nickel oxide.

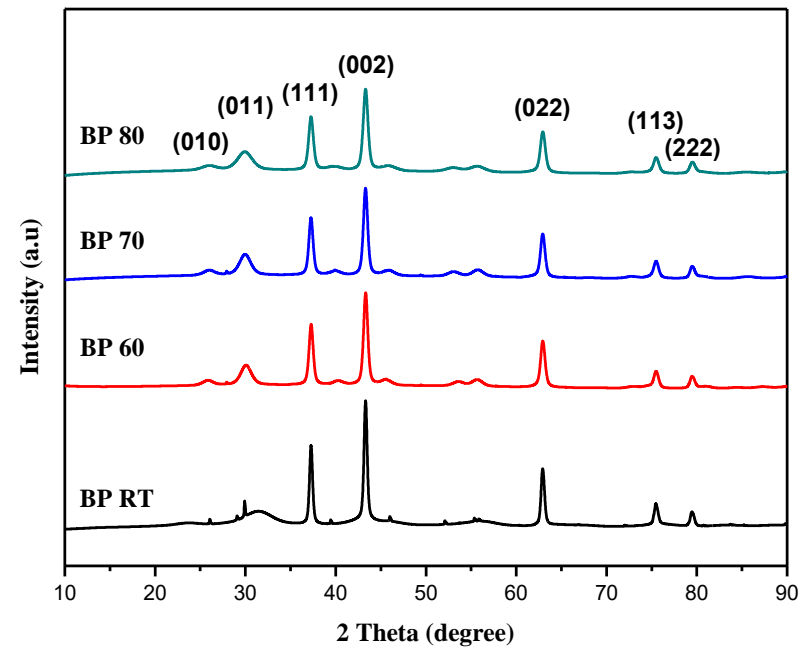

Figure 3. X-ray diffraction pattern for each sample back powder.

Meanwhile, Figure 4 was a magnification of Figure 3 with an angle range of 2 theta $20^{\circ}$ to $40^{\circ}$, where there were differences in the peak pattern in the angle range of 2 theta. In the sample, black powder room temperature can be indicated at magnification with a range of angles that the peak at an angle of 2 theta $26^{\circ}, 29^{\circ}, 30^{\circ}$, and $31^{\circ}$ is the peak of the lanthanum oxide $\left(\mathrm{La}_{2} \mathrm{O}_{3}\right)$ phase. While at an angle of 2 theta $23^{\circ}$ and $31^{\circ}$ was the phase of the lanthanum nickel tetraoxide $\left(\mathrm{La}_{2} \mathrm{NiO}_{4}\right)$. In addition, it was also observed at the diffractogram peaks for black, $60{ }^{\circ} \mathrm{C}, 70{ }^{\circ} \mathrm{C}$, and $80{ }^{\circ} \mathrm{C}$ black powder samples. An identical peak was seen at an angle of 2 theta were $25^{\circ}$ and $30^{\circ}$ which is the phase of lanthanum oxide. While the peak at an angle of 2 theta was $28^{\circ}$, it experiences shrinkage as the temperature of the synthesis rises, which indicates that the angle represents the lanthanum oxide Ht (x-form) phase.

On the other hand, in Figure 5 there was also the formation of peaks in the range of $30^{\circ}$ to $60^{\circ}$, at an angle of $39^{\circ}$ and $44^{\circ}$ observed that there is a paw that shows the lanthanum oxide phase, as the temperature rises, the formation of the peak shows more clearly that the lanthanum phase is formed oxide. Similarly, around the angle of 2 theta $40^{\circ}$ and $45^{\circ}$ also formed a diffractogram peak which showed the development of the phase of lanthanum nickel trioxide at room temperature, but along with the increase in temperature appeared lanthanum oxide phase at the peak at $53^{\circ}$ and $55^{\circ}$.

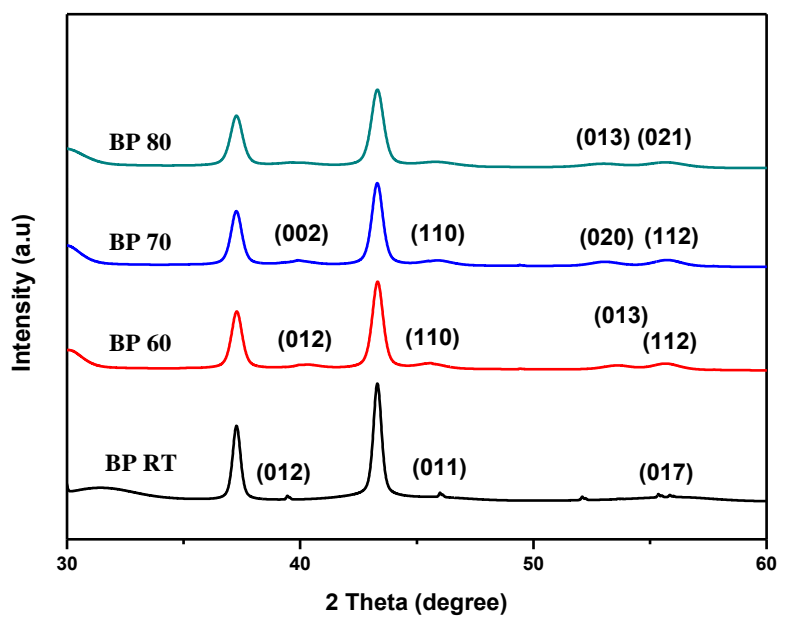

Figure 4. Magnification of diffractogram black powder sample on 20: 30 $-60^{\circ}$.

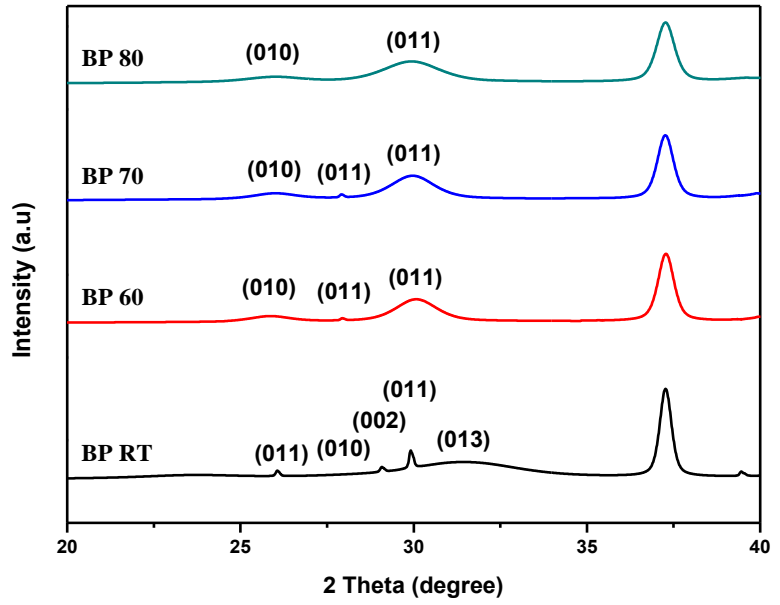

Figure 5. Magnification of diffractogram black powder sample on 2 20 : 20 $-40^{\circ}$.

Judging from the suitability criteria for the profile fitting process based on the literature, the good refinement match is determined by the Weighted Rprofile (Rwp) and goodness of fit (GoF) parameters. Rwp is a weight comparison pattern of the difference between observation patterns with XRD calculations (Speakman, 2012). The ideal value of Rwp is $<10 \%$ 
(Speakman, 2012, Sarwanto and Adi, 2018). While $R_{\text {expected }}$ is a statistical evaluation of noise data on diffractograms. In addition, the parameter of goodness of fit $(\mathrm{GoF})$ or commonly known as Chi-Squared is a ratio of XRD observation patterns that are comparable with expected, with an ideal value of no more than $4 \%$ (Speakman, 2012). The goodness of fit and Rwp factors, as well as the size of the crystals that have been calculated using High Score Plus for each black powder sample, are known respectively in Table 1.

Table 1. Match parameters and crystal size of each black powder sample.

\begin{tabular}{lllcc}
\hline \multirow{2}{*}{ Sample } & \multicolumn{3}{c}{ Uniform Parameter } & \multirow{2}{*}{$\begin{array}{c}\text { Crystal } \\
\text { size }\end{array}$} \\
\cline { 2 - 4 } & $\mathbf{R}_{\text {expected }}$ & $\mathbf{R}_{\mathbf{w p}}$ & $\mathbf{G o F}$ & \\
\hline $\begin{array}{l}\text { Black Powder } \\
\text { Room Temperature }\end{array}$ & 9.1453 & 7.9019 & 1.1902 & 2.0737 \\
Black Powder $60^{\circ} \mathrm{C}$ & 9.0551 & 8.1407 & 1.2738 & 5.0808 \\
Black Powder $70^{\circ} \mathrm{C}$ & 9.1597 & 8.1181 & 1.2420 & 4.3323 \\
Black Powder $80^{\circ} \mathrm{C}$ & 8.9618 & 7.6367 & 1.1468 & 3.4879 \\
\hline
\end{tabular}

From Table 1 it can be concluded that the level of fitting for each black powder sample can be categorized very well according to the literature mentioned earlier. In addition, the results of the analysis of the composition of compounds contained in each black powder sample are listed in Table 2. Table 2 showed that in the black powder room temperature sample, there is a $\mathrm{La}_{2} \mathrm{NiO}_{4}$ phase with a composition of $27.4 \%$.

Table 2. Phase composition contained in the black powder sample.

\begin{tabular}{lllll}
\hline \multirow{2}{*}{ Compound } & \multicolumn{5}{c}{ Composition of Compounds (\%) } \\
\cline { 2 - 5 } & $\mathbf{R . T}$ & $\mathbf{6 0}{ }^{\circ} \mathbf{C}$ & $\mathbf{7 0}^{\circ} \mathbf{C}$ & $\mathbf{8 0}^{\circ} \mathbf{C}$ \\
\hline $\mathrm{La}_{2} \mathrm{NiO}_{4}$ & 27.4 & - & - & - \\
$\mathrm{NiO}$ & 71.3 & 84.6 & 82.0 & 80.7 \\
$\mathrm{La}_{2} \mathrm{O}_{3}$ & 1.3 & 15.3 & 17.8 & 19.3 \\
$\mathrm{La}_{2} \mathrm{O}_{3}-\mathrm{Ht}$ (x-form) & - & 0.1 & 0.2 & - \\
TOTAL & $100 \%$ & $100 \%$ & $100 \%$ & $100 \%$ \\
\hline
\end{tabular}

In each black powder sample $60-80{ }^{\circ} \mathrm{C}$, the $\mathrm{La}_{2} \mathrm{NiO}_{4}$ phase was not formed and tended to show a very dominant $\mathrm{NiO}$ phase and it was observed that with increasing synthesis temperature, $\mathrm{La}_{2} \mathrm{O}_{3}$ phase composition tends to increase.

\section{Scanning Electron Microscopy (SEM) Analysis}

The analysis was carried out with three magnifications, namely 500x, 1000x and 10000x. The sample chamber is vacuum up to $3.54 \times 10^{-4} \mathrm{~Pa}$ for $500 \mathrm{x}$ magnification and $19.4 \times 10^{-2} \mathrm{~Pa}$ for $1000 \mathrm{x}$ and $10000 \mathrm{x}$ magnification, respectively, to ensure that the SEM column is free of air molecules. The SEM was operated with a standard

High Voltage operation parameter of $10 \mathrm{kV}$ with a Spot Size of 2.5 and a Work Distance (WD) of $9.6 \mathrm{~mm}$ for a $60^{\circ} \mathrm{C}$ black powder sample. SEM results for the microstructure test of a $60^{\circ} \mathrm{C}$ black powder sample are shown in Figure 6 taken with a SE detector.
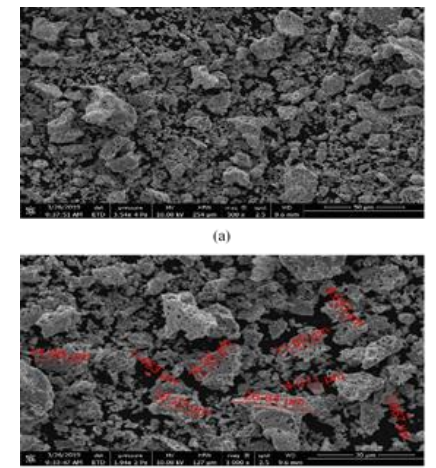

(c)
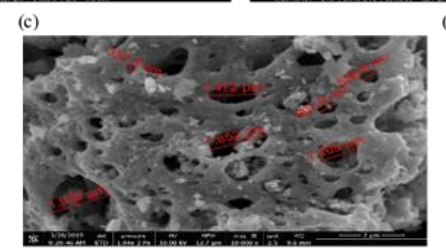

(e)

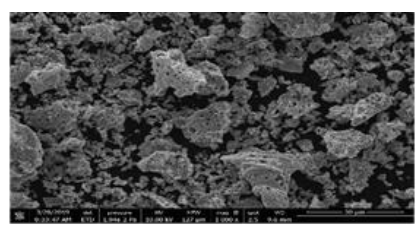

(b)

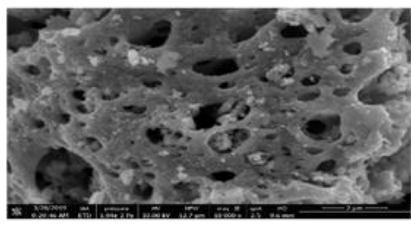

(d)

\section{(1)}

Figure 6. The microstructure of black powder $60^{\circ} \mathrm{C}$ (a) with a magnification of $500 \mathrm{x}$ (b) with a magnification of $1000 \mathrm{x}$ (c) the measurement of molecular samples of black powder and (d) with a magnification of $10000 \mathrm{x}$ (e) measurement of the diameter of the pores formed.

At 500x magnification (Figure 6a), black powder particles are scattered to form small fragments and some are porous chunks. To clarify the observed porous profile, a $1000 x$ magnification was performed. The analysis results with higher magnifications observed that there were agglomerates in the porous lump (Figure 6b). The average particle size observed at 1000x magnification is in the micron range with an average particle size of $11,8093 \mu \mathrm{m}$ (Figure 6c), but it has not provided information about the porosity of the black sample particles the powder. On that basis, the analysis continued with 10000x magnification. In micrographs with a magnification of $10000 x$, it was able to show important information, which was very clearly visible agglomerates contained in the porous lump (Figure 6d) and the size of the pores in the micron order with an average pore diameter of $22.8361 \mu \mathrm{m}$ (Figure 6e).

Then the analysis for the $70{ }^{\circ} \mathrm{C}$ black powder sample is presented in Figure 7. With the SEM operation specifications for the High Voltage operation parameter of $10 \mathrm{kV}$ with Spot Size 2.5 and Work Distance (WD) of $10 \mathrm{~mm}$ for the $70^{\circ} \mathrm{C}$ black powder sample. The sample chamber is vacuum up to $1.30 \times 10^{-4} \mathrm{~Pa}$ for $500 \mathrm{x}$ magnification and $1.41 \times 10^{-4} \mathrm{~Pa}$ for $1000 \mathrm{x}$ magnification and $1.52 \times 10^{-4}$ for $10000 x$ magnification. In micrographs with a magnification of $500 x$, it was seen that in the $70^{\circ} \mathrm{C}$ black powder sample, the particles in the sample 
were scattered with mostly dominated by lumps (Figure 7a). Then, it was clear that the lump had pores on each of the lumps (Figure 7b) with an average size of the lumps of 20,89 $\mu \mathrm{m}$ (Figure 16c). In addition, at 10,000x magnification (Figure 7d), it was observed that there were agglomerates with an average of $0,5632 \mu \mathrm{m}$ and an average pore diameter of $0,7508 \mu \mathrm{m}$ (Figure $7 \mathrm{e}$ ).
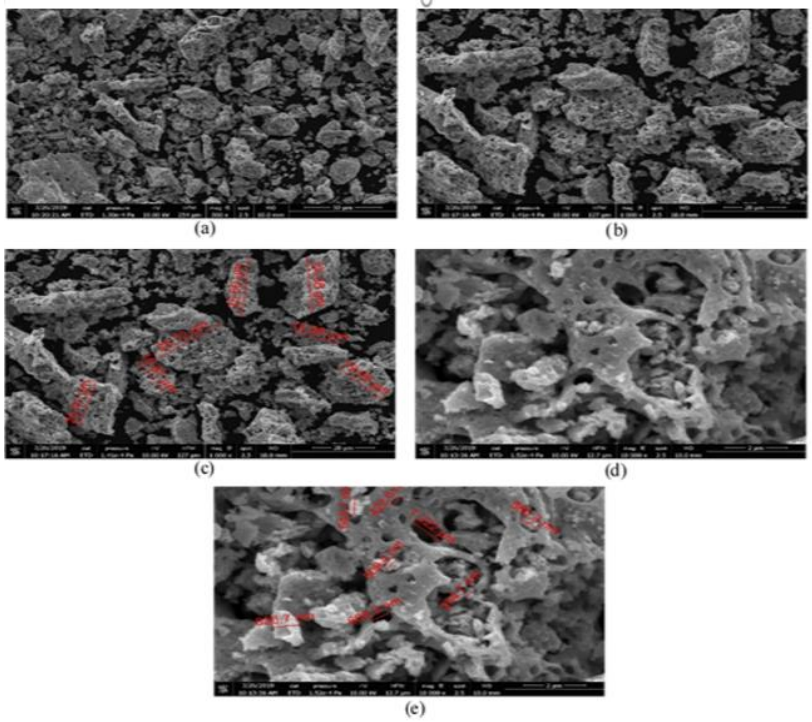

Figure 7. The microstructure of black powder $70{ }^{\circ} \mathrm{C}$ (a) with a magnification of $500 \mathrm{x}$ (b) with a magnification of $1000 \mathrm{x}$ (c) the measurement of molecular samples of black powder and (d) with a magnification of $10000 \mathrm{x}$ (e) measurement of the diameter of the pores formed.
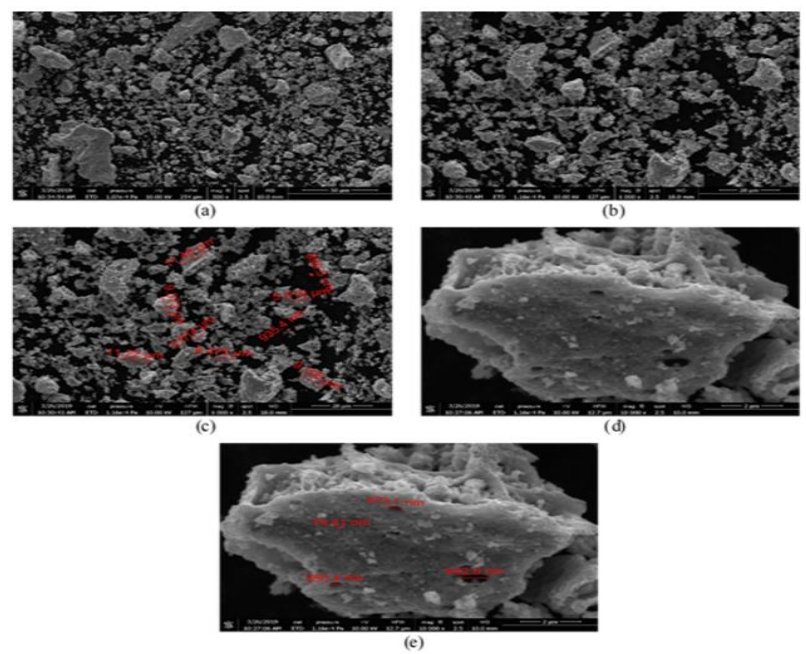

(d)

Figure 8. The microstructure of black powder $80^{\circ} \mathrm{C}$ (a) with a magnification of 500x (b) with a magnification of 1000x (c) molecular measurements of the black powder sample and (d) with a magnification of $10000 \mathrm{x}$ (e) measurement of the diameter of the pores formed.

After that, the $80{ }^{\circ} \mathrm{C}$ black powder micrograph sample was shown in Figure 8. The micrograph is obtained with a High Voltage operation parameter of 10 $\mathrm{kV}$ with a Spot Size of 2.5 and a Work Distance (WD) of $10 \mathrm{~mm}$. The sample chamber is vacuum up to
$1.07 \times 10^{-4} \mathrm{~Pa}$ for $500 \mathrm{x}$ magnification and $1.16 \times 10^{-4} \mathrm{~Pa}$ for 1000x and 10000x magnification, respectively.

In Figure 8, it appears that the $80^{\circ} \mathrm{C}$ black powder sample does not look too many lumps that appear and most are shaped granules. Then a magnification of $1,000 \mathrm{x}$ was carried out so that it was observed that the particle structure of the black powder sample tended to have pores that were slightly unlike the micrographs of the black powder samples $60{ }^{\circ} \mathrm{C}$ and $70{ }^{\circ} \mathrm{C}$. In addition, information was obtained in the form of particle size with an average of $6.7447 \mu \mathrm{m}$ (Figure 8c). In micrographs with 10,000x magnification (Figure 8d and $8 \mathrm{e})$, it is known that in the black powder sample $80{ }^{\circ} \mathrm{C}$, the shaft/pores of the particles shrink and tend to be more solid with an average shaft/pores of $0.4676 \mu \mathrm{m}$ tend to be smaller compared to the black powder samples in Figure 6 and Figure 7.

The morphology of black, $60{ }^{\circ} \mathrm{C}, 70{ }^{\circ} \mathrm{C}$, and $80{ }^{\circ} \mathrm{C}$ samples in the Figure $6 \mathrm{~d}$, Figure $7 \mathrm{~d}$, and Figure $8 \mathrm{~d}$ with dark colours that appear to be more dominant in each sample is a constituent element with a low atomic number. In contrast, the bright colours seen in agglomerates are constituent elements that have high atomic numbers (Tutu et al., 2015). So it can be concluded that the dark side of the sample contains $\mathrm{Ni}$ atoms and agglomerates that appear to be representations of $\mathrm{La}$ atoms.

From the three micrographs, it appears that the high surface area was affected by the type of glycine fuel which causes rapid thermal decomposition and excessive gas development, resulting in greater porosity and crystal size (Deganello and Tyagi, 2018; Fathi et al., 2017). It is also known that the influence of temperature variations in the synthesis can affect the porosity and size of the crystal.

\section{Energy Dispersive X-Ray Spectroscopy (EDS) Analysis}

EDS analysis on each black powder sample obtained from the Solution Combustion Synthesis (SCS) synthesis method was carried out to determine the composition of the elements in the sample. The results of the EDS analysis for each black powder sample are presented in Figures 9, Figure 10 and Figure 11.

Based on the results of the EDS analysis in Figure 9, it was known that the microstructure for black powder samples with a temperature variation of $60^{\circ} \mathrm{C}$ contains the composition of the elements $\mathrm{O}, \mathrm{Ni}$, and $\mathrm{La}$ at each observation point with the relative percent mass of the element at the first observation point (Figure 9a) for $\mathrm{Ni}$ of $57.8 \%$, element $\mathrm{La}$ is $26.6 \%$, and element $\mathrm{O}$ is $15.6 \%$. Then at the second observation point (Figure 9b) shows the relative mass of each element, each for $\mathrm{Ni}$ is $51.4 \%$, element $\mathrm{La}$ is $27.8 \%$, and element O is $20.9 \%$. Similarly, the relative mass at the third observation point (Figure 
9c) for each element is as follows for $\mathrm{Ni}$ of $54.1 \%$, element $\mathrm{La}$ for $26.3 \%$, and element $\mathrm{O}$ for $19.6 \%$.

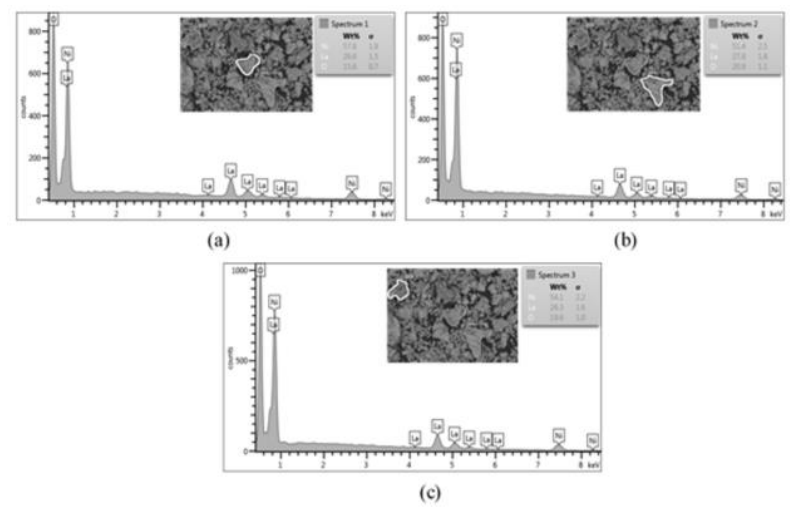

Figure 9. The EDS spectrum and composition of the $60{ }^{\circ} \mathrm{C}$ black powder sample resulting from the SCS synthesis method with three observation points. (a) EDS analysis at the first observation point. (b) EDS analysis at the second observation point. (c) EDS analysis at the third observation point.

In addition, Figure 10 shows the EDS spectrum for black powder samples with a temperature variation of 70 ${ }^{\circ} \mathrm{C}$ containing the composition of the elements $\mathrm{Ni}$, La and $\mathrm{O}$. with each relative mass of the element at the first observation point (Figure 10a), namely Ni of 56.3\%, element $\mathrm{La}$ is $24.8 \%$, and element $\mathrm{O}$ is $18.9 \%$. Furthermore, at the second observation point (Figure $10 \mathrm{~b}$ ), it was found that the relative mass of each element, including $\mathrm{Ni}$ was $55.2 \%$, La was $27.9 \%$ and $\mathrm{O}$ was $16.9 \%$. Then the observation at the third point (Figure 10c) obtained the relative mass composition of each element, including $\mathrm{Ni}$ of $61.2 \%$, La of $27.1 \%$ and $\mathrm{O}$ of $11.7 \%$.

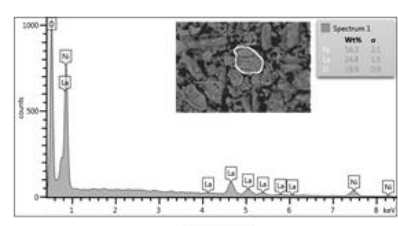

(a)

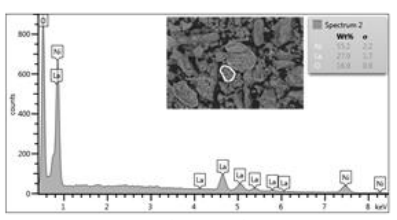

(b)

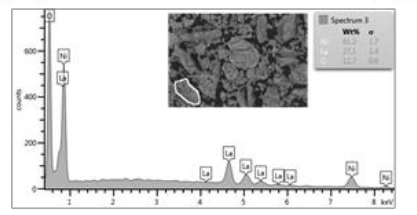

(c)

Figure 10. The EDS spectrum and the composition of the $70^{\circ} \mathrm{C}$ black powder sample resulting from the SCS synthesis method with three observation points. (a) EDS analysis at the first observation point. (b) EDS analysis at the second observation point. (c) EDS analysis at the third observation point.

On the temperature of $80{ }^{\circ} \mathrm{C}$, the black powder sample presented in Figure 11 showing three observation points on the surface of the black powder sample, the results show that there are elemental compositions as follows $\mathrm{Ni}, \mathrm{La}$, and $\mathrm{O}$ with the relative mass content of each element at the first observation point (Figure 11a) $\mathrm{Ni}$ at $57.1 \%$, element $\mathrm{La}$ at $25.1 \%$, and element $\mathrm{O}$ at $17.8 \%$. Whereas at the second observation point (Figure 11b), each element's relative mass composition as follows on the $\mathrm{Ni}$ element is $56.4 \%$, La element is $26.9 \%$, and element $\mathrm{O}$ is $16.7 \%$. At the last observation point (Figure 11c), the relative mass composition of each element is $57.2 \%$ for the $\mathrm{Ni}$ element, $25.2 \%$ for the La element, and $17.6 \%$ for the $\mathrm{O}$ element.

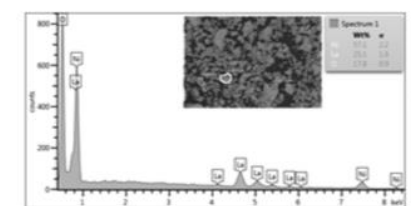

(a)

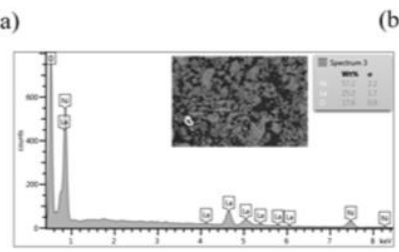

(c)
Figure 11. The EDS spectrum and the composition of the $80^{\circ} \mathrm{C}$ black powder sample resulting from the SCS synthesis method with three observation points. (a) EDS analysis at the first observation point. (b) EDS analysis at the second observation point. (c) EDS analysis at the third observation point.

The following is summarized in Table 3 results of the analysis of Energy Dispersive X-ray Spectroscopy (EDS).

Table 3. EDS test analysis results for each black powder sample.

\begin{tabular}{ccccc}
\hline \multirow{2}{*}{ Observation } & \multicolumn{5}{c}{ Element (\%) } \\
\cline { 2 - 5 } & Ni & La & O & Total \\
\hline \multicolumn{5}{c}{ Black Powder 60 } \\
Point 1 & 57.8 & 26.6 & 15.6 & 100 \\
Point 2 & 51.4 & 27.8 & 20.9 & 100 \\
Point 3 & 54.1 & 26.3 & 19.6 & 100 \\
\hline \multicolumn{5}{c}{ Black Powder 70 } \\
\hline Point 1 & 56.3 & 24.8 & 18.9 & 100 \\
Point 2 & 51.4 & 27.8 & 20.9 & 100 \\
Point 3 & 61.2 & 27.1 & 11.7 & 100 \\
\hline \multicolumn{7}{c}{ Black Powder 80 } \\
\hline Point 1 & 57.1 & 25.1 & 17.8 & 100 \\
Point 2 & 56.4 & 26.9 & 16.7 & 100 \\
Point 3 & 57.2 & 25.2 & 17.6 & 100 \\
\hline
\end{tabular}

It appears that there was three percent by weight of the dominant atom contained in each black powder sample. In addition, it was confirmed that the nickel element has a composition of atomic weight that is greater and most dominant for each black powder 
sample and the element $\mathrm{La}$ is the lowest element. Then in the results, it can be concluded that the EDS test is EDS-Oxide seen in the presence of element O. So that due to the presence of element $\mathrm{O}$, oxidation occurs in the elements of $\mathrm{Ni}$ and $\mathrm{La}$ and the compound element is formed which is $\mathrm{Ni}$ into $\mathrm{NiO}$ (Nickel Oxide), and the element It becomes $\mathrm{La}_{2} \mathrm{O}_{3}$ (Setiadi, 2018). Therefore, this result is in accordance with the compounds' composition results where the phases formed are Nickel Oxide and Lanthanum Oxide and the Nickel Oxide compound phase has a very dominant compound composition. This data supports data from XRD where no other peaks are found as impurities outside the reactants used.

\section{Fourier Transform Infrared (FTIR) Analysis}

The Fourier Transform Infrared spectrum for the three black powder samples synthesized using the solution combustion synthesis (SCS) method with temperature variations (Room Temperature, 60, 70, and $80^{\circ} \mathrm{C}$ ) and the forming material is shown in Figure 12.

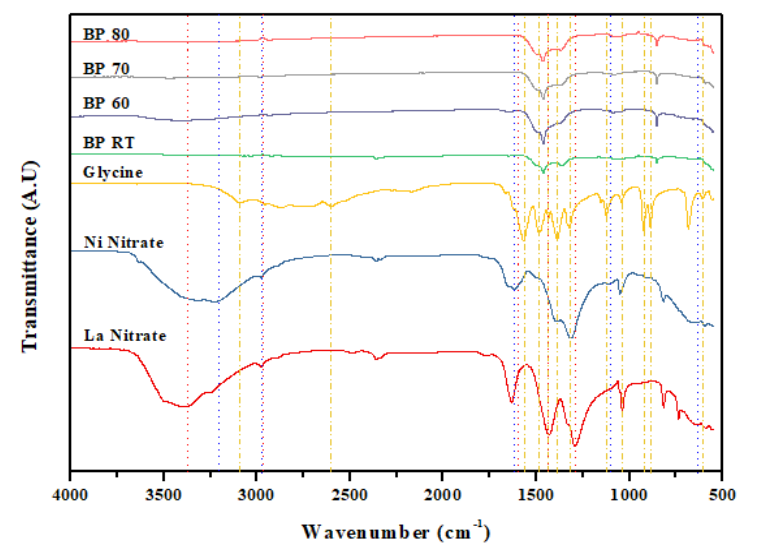

Figure 12. FTIR spectrum of each black powder sample with its raw material.

The Fourier Transform Infrared Spectrum (FTIR) of the sample is then compared with each of its constituent materials. So it is known that each chemical bonding group is formed and disappears in the black powder sample. Based on the analysis that has been done, there are several peaks in the spectrum of Lanthanum Nitrate Hexahydrate $\left(\mathrm{La} \quad\left(\mathrm{NO}_{3}\right)_{3} \cdot 6 \mathrm{H}_{2} \mathrm{O}\right)$ which have been identified in Table 4. In the FTIR spectrum of the material forming the Lanthanum Nitrate Hexahydrate, it is known that in the forming material, several chemical bonding groups appear, which is found in the wavenumber $3200-3550 \mathrm{~cm}^{-1}$, which indicates the existence of $\mathrm{O}-\mathrm{H}$ stretching vibrations with strong and wide spectrum characteristics. The $\mathrm{O}-\mathrm{H}$ bonding group shows that the sample contains water molecules (Nedumkallel et al., 2014).
Table 4. FTIR spectrum of lanthanum nitrate hexahydrate, nickel nitrate hexahydrate, and glycine.

\begin{tabular}{|c|c|}
\hline Wavenumber $\left(\mathrm{cm}^{-1}\right)$ & Assignment of vibrations \\
\hline \multicolumn{2}{|c|}{$\begin{array}{c}\mathrm{La}\left(\mathrm{NO}_{3}\right)_{3} .6 \mathrm{H}_{2} \mathrm{O} \\
\end{array}$} \\
\hline 3200-3550 & $\mathrm{O}-\mathrm{H}$ bending \\
\hline 2.980 & $\mathrm{C}-\mathrm{H}$ bending, medium \\
\hline $1293-1434$ & $\mathrm{NO}_{3}$ asymmetric raw material \\
\hline 1368-1464 & $\begin{array}{l}\mathrm{NO}_{3} \text { bonding asymmetric sample } \\
\text { black powder. }\end{array}$ \\
\hline $\begin{array}{l}1649,1646 \text {, and } \\
1640\end{array}$ & $\begin{array}{l}\mathrm{N}=\mathrm{O} \text { bonding on black powder } \\
\text { samples } 60,70 \text {, and } 80^{\circ} \mathrm{C}\end{array}$ \\
\hline $415-423$ & La-O group \\
\hline \multicolumn{2}{|r|}{$\mathrm{Ni}\left(\mathrm{NO}_{2}\right)_{3} .6 \mathrm{H}_{2} \mathrm{O}$} \\
\hline 3550-3200 & $\mathrm{O}-\mathrm{H}$ bonding \\
\hline 1619 & Hydroxyl group \\
\hline 1110 & Carbonate group \\
\hline 2980 & C-H bonding \\
\hline 633 & Ni-O-H bonding \\
\hline $447-552$ & $\mathrm{Ni}-\mathrm{O}$ bonding \\
\hline \multicolumn{2}{|r|}{ Glycine } \\
\hline 607, 1389, and 1570 & Carboxylate group $\mathrm{COO}^{-}$ \\
\hline 1389 & $\begin{array}{l}\text { Carboxylate group symmetrical } \\
\text { COO- }^{-}\end{array}$ \\
\hline $1125,1489,2600$ & Ammonium group $\mathrm{NH}_{3}{ }^{+}$ \\
\hline 2600 and 3090 & Ammonium group $\mathrm{NH}_{3}{ }^{+}$ \\
\hline 889 and 1044 & $\begin{array}{l}\mathrm{CCN} \text { asymmetric and simetric } \\
\text { bonding }\end{array}$ \\
\hline 926 & $\mathrm{CH}_{2}$ rocking \\
\hline 1322 & $\mathrm{CH}_{2}$ twisting \\
\hline 1435 & $\mathrm{CH}_{2}$ bonding \\
\hline
\end{tabular}

On Tabel 4, peaks around $\sim 2980 \mathrm{~cm}^{-1}$ were observed due to vibration stretching of the $\mathrm{C}-\mathrm{H}$ bond with the characteristic of stretching with medium intensity (Kumari et al., 2015). In addition, it was observed that there was $\mathrm{NO}_{3}$ stretching asimeter which appeared at $1293-1434 \mathrm{~cm}^{-1}$ in the forming material and at 1368$1464 \mathrm{~cm}^{-1}$ in each black powder sample. This shows the change in the $\mathrm{NO}_{3}$ stretching wave number due to the possibility of constant changes in the force and dipole moment of the $\mathrm{NO}_{3}$ group vibrations (Trivedi et al., 2015). The characteristic vibration peaks for stretching $\mathrm{N}=\mathrm{O}$ also appear at 1649, 1646, and $1640 \mathrm{~cm}^{-1}$ respectively in black powder samples 60,70 , and $80^{\circ} \mathrm{C}$ which were previously shifted at wave number $1634 \mathrm{~cm}$ 1 in the forming material (Lanthanum Nitrate Hexahydrate). Whereas La-O chemical bonding group based on literature shows the wavenumber $415-423 \mathrm{~cm}^{-1}$ (Radev et al., 2008). However, Figure 12 only covers the range of $500-4000 \mathrm{~cm}^{-1}$.

Then in the infrared spectrum of Ni nickel nitrate hexahydrate $\left(\mathrm{NO}_{3}\right)_{2} \cdot 6 \mathrm{H}_{2} \mathrm{O}$ material, it was seen that several peaks were formed, including the wave number 
$3550-3200 \mathrm{~cm}^{-1}$ which was an $\mathrm{O}-\mathrm{H}$ bond and around the wave number $1619 \mathrm{~cm}^{-1}$ associated with the group hydroxyl (Rahdar et al., 2015). However, stretching vibration modes of $\mathrm{H}-\mathrm{O}-\mathrm{H}$ around the wave number $1658 \mathrm{~cm}^{-1}$ does not appear in the spectrum pattern (Segawa et al., 2015). When compared with the infrared spectrum in the black powder sample, it was observed that the stretching of the $\mathrm{O}-\mathrm{H}$ bond was reduced, which was caused by the drying and calcination treatment process carried out on the black powder sample. The absorption of the wavenumber $1110 \mathrm{~cm}^{-1}$ shows the presence of carbonates and bonds at around $2980 \mathrm{~cm}^{-1}$ according to the $\mathrm{C}-\mathrm{H}$ stretch (Rahdar et al., 2015). The $\mathrm{Ni}-\mathrm{O}-\mathrm{H}$ stretching bond was observed at wave number $633 \mathrm{~cm}^{-1}$, but the absorption of the wavenumber at 447 $552 \mathrm{~cm}^{-1}$ associated with the Ni-O bond stretching was not observed, due to the range of wave numbers which only ranged from 4000 to $500 \mathrm{~cm}^{-1}$ (Nedumkallel et al., 2014, Rahdar et al., 2015).

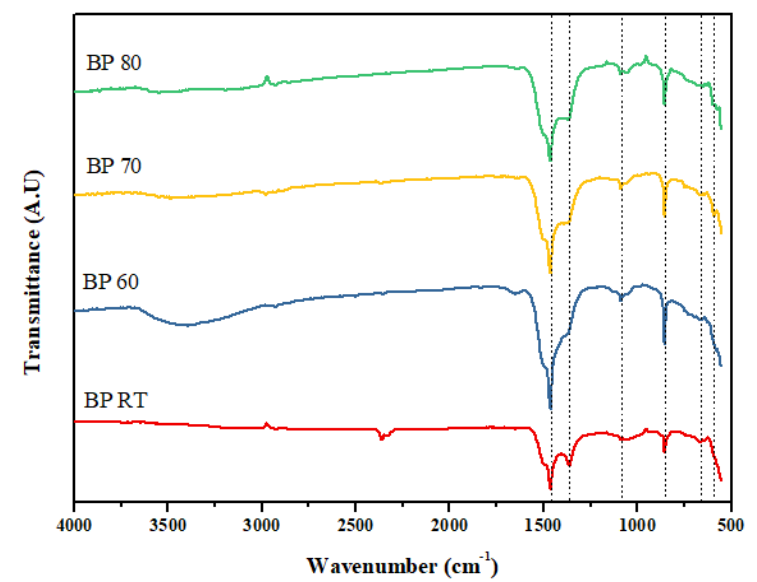

Figure 13. The infrared spectrum of a black powder sample has been tested with FTIR.

In the last infrared spectrum, which is glycine material, it was known that in this comparison, there was a free glycine carboxylic group (COO) which is usually observed at 607,1414 and $1605 \mathrm{~cm}^{-1}$. As in the case of glycine in this forming material, the peak shifts to 607 , 1388, and $1570 \mathrm{~cm}^{-1}$ (Ahamed et al., 2013, Sankar et al., 2010). Observed at the peak of $1389 \mathrm{~cm}^{-1}$, there was a stretching of the COO- symmetric carboxylate group according to the reported value of literature (Azhagan \& Ganesan, 2013). In addition, peaks in the wavenumbers 1125, 1489, $2600 \mathrm{~cm}^{-1}$ are shifted from the values presented in the literature at wavenumbers 1133, 1507, and $2614 \mathrm{~cm}^{-1}$ of the ammonium group $\left(\mathrm{NH}^{3+}\right.$ ) (Ahamed et al., 2013). Whereas the absorption peak with wavenumbers of 2600 and $3090 \mathrm{~cm}^{-1}$ was indicated as stretching $\mathrm{NH}^{3+}$ (Peter \& Ramasamy, 2010). The peaks were observed at wavenumbers 889 and $1044 \mathrm{~cm}^{-1}$ which are asymmetric and symmetric CCN stretches. Whereas the peaks observed at wavenumbers 926, 1322, and $1435 \mathrm{~cm}^{-1}$ are respectively $\mathrm{CH}_{2}$ rocking, $\mathrm{CH}_{2}$ twisting, and $\mathrm{CH}_{2}$ bending (Azhagan \& Ganesan, 2013). So from the analysis of the three row materials, it was found that there were several new absorption peaks that appeared, and some absorptive peaks were lost as shown in Figure 13.

From the peak of the absorption that appeared based on Merck - Infrared Spectrum Table and Chart, among them, there were around wavenumbers 1464 and 1462 $\mathrm{cm}^{-1}$ in each black powder sample showing the presence of $\mathrm{C}-\mathrm{H}$ bending bonds with medium intensity and indicated as a class of alkane compounds. Then, there is also the absorption peak at the wavenumbers 1390 and $1391 \mathrm{~cm}^{-1}$ in the black powder samples $60{ }^{\circ} \mathrm{C}$ and $70^{\circ} \mathrm{C}$ which were indicated as $\mathrm{C}-\mathrm{H}$ bending bonds with medium intensity and were observed as an aldehyde class. A strong C-O stretching bond was also observed at the absorption peak around the wave number 1087 $\mathrm{cm}^{-1}$ with indications belonging to the secondary alcohol class. These are summarized in Table 5.

Table 5. FTIR spectrum of black powder sample.

\begin{tabular}{ll}
\hline Wavenumber $\left(\mathbf{c m}^{-1}\right)$ & Assignment of vibrations \\
\hline $\mathbf{1 4 6 4}$ & Black Powder 60 \\
\hline $\mathbf{1 3 9 0}$ & C-H Bending, medium, alkane \\
\hline $\mathbf{1 3 6 8}$ & C-H Bending, medium, aldehyde \\
\hline $\mathbf{1 0 8 7}$ & - \\
\hline $\mathbf{8 5 4}$ & C-O Stretching, Strong, Secondary \\
\hline $\mathbf{6 4 9}$ & Alcohol \\
\hline \multicolumn{2}{|c}{-} \\
\hline $\mathbf{1 4 6 2}$ & - \\
\hline $\mathbf{1 3 9 1}$ & Black Powder 70 \\
\hline $\mathbf{1 0 8 7}$ & C-H Bending, medium, alkane \\
\hline $\mathbf{8 5 4}$ & C-H Bending, medium, aldehyde \\
\hline $\mathbf{6 5 6}$ & C-O Stretching, Strong, Secondary \\
\hline $\mathbf{5 9 1}$ & Alcohol \\
\hline & - \\
\hline $\mathbf{1 4 6 2}$ & - \\
\hline $\mathbf{1 0 8 7}$ & - \\
\hline $\mathbf{8 5 4}$ & Black Powder 80 \\
\hline $\mathbf{6 4 9}$ & C-H Bending, medium, alkane \\
\hline $\mathbf{5 7 9}$ & C-O Stretching, Strong, Secondary \\
\hline & Alcohol \\
\hline & - \\
\hline
\end{tabular}

It was observed that all of the three black powder samples as a whole $\mathrm{O}-\mathrm{H}$ stretching bond groups were reduced. This can be influenced by the drying process (evaporate process). So the $\mathrm{O}-\mathrm{H}$ stretching bonds that indicate the element $\mathrm{H}_{2} \mathrm{O}$ evaporates at their boiling temperature. While the $\mathrm{C}-\mathrm{O}$ and $\mathrm{C}-\mathrm{H}$ bonds in the infrared spectrum indicate that there are still organic elements owned by glycine, which acts as fuel, so it can be concluded that the temperature during the calcination 
process was still not optimal. Not yet the optimal formation of this phase refers to the calcination process in accordance with its purpose, so that the optimal temperature needed in the calcination process to get the phase in accordance with the reaction equation from the reference in this study.

\section{CONCLUSION}

Based on the results of the research that had been carried out, some conclusions can be taken as follows:

1. In this study, black powder $\left(\mathrm{La}_{2} \mathrm{NiO}_{4}\right)$ material with a variation of synthesis temperature of 60,70 , and 80 ${ }^{\circ} \mathrm{C}$ with the Solution Combustion Synthesis method produces a sample weight of 0.5 grams 4 times the synthesis process so that each sample is obtained by 2 grams. In addition, it is known that there is heavy evaporation of $96.11 \%$ of the total weight of the synthesized precursors.

2. X-Ray Diffraction analysis results from each sample indicated that there were several phases contained in the black powder, namely Nickel Oxide, Lanthanum Oxide, and Lanthanum Oxide $\mathrm{Ht}$ (x-form). By varying the synthesis temperature, the composition of the Lanthanum Nickel Oxide $\left(\mathrm{La}_{2} \mathrm{NiO}_{4}\right)$ phase disappears and makes a tendency for chemical reactions to help the two dominant phases, namely the Nickel and the Lanthanum oxide phase. This is supported by the results of Scanning Electron Microscopy with micrographs that appear dominant with a dark colour that shows a low atomic number indicating the element $\mathrm{Ni}$ and light colours on agglomerates that show a high atomic number which indicates the element $\mathrm{La}$. In addition, the phase formation previously carried out by XRD analysis is strengthened through EDS analysis where the results show that the graph is EDS-Oxide, seen in the presence of element $\mathrm{O}$. So because of the presence of element $\mathrm{O}$, oxidation occurs in the elements $\mathrm{Ni}$ and $\mathrm{La}$ and a compound element occurs formed namely $\mathrm{Ni}$ to $\mathrm{NiO}$ (Nickel Oxide) and the element $\mathrm{La}$ to $\mathrm{La}_{2} \mathrm{O}_{3}$ (lanthanum Oxide). In addition, Fourier Transform Infrared analysis shows that there are still $\mathrm{C}-\mathrm{O}$ and $\mathrm{C}-\mathrm{H}$ bonds which are bonds of glycine surfactants, thus indicating that the process of phase formation in accordance with the reference reaction is not optimal. Then we need an optimal calcination temperature.

Acknowledgments: We are thanks to the WP Team Rare Earth Metal Prototypes at the Center for Material Technology-Agency for the Assessment and Application of Technology for providing all the required laboratory facilities.
Conflict of interest: The author declares that there are no conflicts of interest concerning the publication of this article.

\section{REFERENCES}

Adziimaa A. F., Risanti D. D., and Mawarni J. (2013). Synthesis of Sodium Silicate from Lapindo Mud as Corrosion Inhibitor. J. Tek. ITS, 2(2), 384-389.

Ahamed, S. Z. A., Dillip, G. R., Raghavaiah, P., Mallikarjuna, K., \& Raju, B. D. P. (2013). Spectroscopic and thermal studies of $\gamma$-glycine crystal grown from potassium bromide for optoelectronic applications. Arabian Journal of Chemistry, 6(4), 429-433.

Aruna, S. T. (2017). Solution combustion synthesis. In Concise encyclopedia of self-propagating high-temperature synthesis (pp. 344-346). Elsevier.

Azhagan, S. A. C., \& Ganesan, S. (2013). Crystal growth, structural, optical, thermal and NLO studies of $\gamma$-glycine single crystals. Optik, 124(23), 6456-6460.

Chang, S., Young, K. H., Nei, J., \& Fierro, C. (2016). Reviews on the US Patents regarding nickel/metal hydride batteries. Batteries, 2(2), 10.

Chang, S., Young, K. H., \& Lien, Y. L. (2017). Reviews of European Patents on Nickel/Metal Hydride Batteries. Batteries, 3(3), 25.

Deganello, F., \& Tyagi, A. K. (2018). Solution combustion synthesis, energy and environment: Best parameters for better materials. Progress in Crystal Growth and Characterization of Materials, 64(2), 23-61.

Fathi, H., Masoudpanah, S. M., Alamolhoda, S., \& Parnianfar, H. (2017). Effect of fuel type on the microstructure and magnetic properties of solution combusted $\mathrm{Fe} 3 \mathrm{O} 4$ powders. Ceramics International, 43(10), 7448-7453.

González-Cortés, S. L., \& Imbert, F. E. (2013). Fundamentals, properties and applications of solid catalysts prepared by solution combustion synthesis (SCS). Applied Catalysis A: General, 452, 117-131.

Haryadi, H. (2017). Analysis of Indonesia's Iron Sand and Nickel Ore Resources Balance. Journal Mineral Technology dan Batubara. 13 (2), 153-169.

Inatadon, N. F., Abdurrachman, M., and Aziz, M. (2015). Geology and Study of Rare Earth Metals Botor Peanut Area and Surrounding Areas, Badau District, Belitung Regency, Province of Bangka Belitung Islands. Proceeding, National Seminar on Earth Ke-8. 744-753.

Khina, B. B. (2010). Combustion Synthesis of Advanced Materials. New York: Nova Science Publishers, Inc.

Kumari, A., Soni, A. K., Dey, R., and Rai, V. K. (2015). White Light Emission and Optical Heating in $\mathrm{Er}-\mathrm{Tm}-\mathrm{Yb}$ Codoped La2O3 Phosphor. J. Disp. Technol., 12(1), 99-105.

Latif, C., Triwikantoro, and Munasir. (2014). Effect of Calcination Temperature Variation on Silica Structure. $J$. Sains dan Seni ITS, 3(1). 4-7.

Li, M. M., Yang, C. C., Wang, C. C., Wen, Z., Zhu, Y. F., Zhao, M., ... \& Jiang, Q. (2016). Design of hydrogen storage alloys/nanoporous metals hybrid electrodes for nickel-metal hydride batteries. Scientific reports, 6(1), 1-10. 
Liu, W., \& Aguey-Zinsou, K. F. (2016). Low temperature synthesis of LaNi5 nanoparticles for hydrogen storage. International Journal of Hydrogen Energy, 41(3), 1679-1687.

Manukyan, K. V. (2017). Solution combustion synthesis of catalysts. In Concise Encyclopedia of Self-Propagating HighTemperature Synthesis (pp. 347-348). Elsevier.

Nedumkallel, A. S., Sabu, B., and Varghese, T. (2014). Effect of Calcination Temperature On The Structural and Optical Properties. Nanosyst. PHYSICS, Chem. Math., 5(3). 441-449.

Ouchi, T., Young, K. H., \& Moghe, D. (2016). Reviews on the Japanese patent applications regarding nickel/metal hydride batteries. Batteries, 2(3), 21.

Patil, K. C., Aruna, S. T., \& Ekambaram, S. (1997). Combustion synthesis. Current opinion in solid state and materials science, 2(2), 158-165.

Peter, M. E., \& Ramasamy, P. (2010). Growth of gamma glycine crystal and its characterisation. Spectrochimica Acta Part A: Molecular and Biomolecular Spectroscopy, 75(5), 1417-1421.

Prasetiyo, P. (2008). Utilization of Indonesia's Nickel Ore Potential at Present and Future. Metallurgical Magazine, 23 (1).

Radev, L., Pavlova, L., Samuneva, B., Kashchieva, E., Mihailova, I., Zaharescu, M., ... \& Predoana, L. (2008). Sol-gel synthesis and structure of La2O3-CoO-SiO2 powders. Processing and Application of Ceramics, 2(2), 103-108.

Rahdar, A., Aliahmad, M., and Azizi, Y. (2015). NiO Nanoparticles: Synthesis and Characterization," $J$. Nanostructures, 5, 145-151.

Sankar, S., Manikandan, M. R., Ram, S. G., Mahalingam, T., \& Ravi, G. (2010). Gel growth of $\alpha$ and $\gamma$ glycine and their characterization. Journal of crystal growth,312(19), 27292733.

Sarwanto, Y. and Adi, W.A. (2018). Crystallographic Structure and Magnetic Properties of Pseudobrookite Fe2-X NiX TiO5 System $(\mathrm{x}=0,0.1,0.2,0.3,0.5$ and 1). Indones. J. Mater. Sci., 19(2). 47-53.
Segawa, T., Kawaguchi, K., Ishii, K., Suzuki, M., Arimitsu, N., Yoshida, H., \& Fukui, K. (2015). Nickel oxide powder synthesis from aqueous solution of nickel nitrate hexahydrate by a microwave denitration method. Advanced Powder Technology, 26(3), 983-990.

Setiadi, H. (2018). Analysis of Micro Structure and Mechanical Properties of NiCr-Al Coating Formed by Sputtering Method on Steel 40. Universitas Muhammadiah Surakarta.

Speakman, S. A. (2012). Profile Fitting for Analysis of XRPD Data using HighScore Plus v3. Massachusetts Institute of Technology, Cambridge MA.

Trivedi, M. K., Branton, A., Trivedi, D., Nayak, G., Bairwa, K., \& Jana, S. (2015). Spectroscopic characterization of disodium hydrogen orthophosphate and sodium nitrate after biofield treatment. Chromatography Separation Techniques, 6(5).

Tutu, R., Subaer, and Usman. (2015). Analysis study of characterization and microstructure of sulili hot spring sediment in pinrang district. J. Sains dan Pendidik. Fis., 2, 192-201.

Virdhian, S. and Afrilinda, E. (2014). Characterization of Soil Minerals Rarely Follow-Up Tin and Potential Development of Soil-Based Industry Rare Earth. J. Met. Indonesia, 36 (2). 61.

Xanthopoulou, G., Thoda, O., Metaxa, E. D., Vekinis, G., \& Chroneos, A. (2017). Influence of atomic structure on the nano-nickel-based catalyst activity produced by solution combustion synthesis in the hydrogenation of maleic acid. Journal of Catalysis, 348, 9-21.

Xanthopoulou, G., Thoda, O., Roslyakov, S., Steinman, A., Kovalev, D., Levashov, E., ... \& Chroneos, A. (2018). Solution combustion synthesis of nano-catalysts with a hierarchical structure. Journal of catalysis, 364, 112-124.

Young, K. H., Cai, X., \& Chang, S. (2017). Reviews on Chinese Patents regarding the nickel/metal hydride battery. Batteries, 3(3), 24. 
THIS PAGE INTENTIONALLY LEFT BLANK 